\title{
IMPACT OF MAGNETIC FIELD INTERFERENCE IN THE SNS RING*
}

\author{
Y. Papaphilippou ${ }^{\dagger}$, Y. Y. Lee and W. Meng \\ Brookhaven National Laboratory, Upton, NY 11973, USA
}

\section{Abstract}

The modest size of the SNS accumulator ring and the use of short, large aperture magnets makes unavoidable the overlapping between the magnetic end fields of the quadrupoles with the adjacent multipole correctors. This interference effect can be quantified through magnetic field simulations and measurements. The impact to the beam dynamics is finally discussed.

\section{THE SNS HALF-CELL ASSEMBLY}

The Spallation Neutron Source (SNS) ring lattice is a hybrid design [1] consisting of four identical arcs and special function straight sections. The arc has a FODO structure, with four cells (eight quadrupoles and dipoles), whereas the straight sections are occupied by two quadrupole doublets. An additional quadrupole is being located between the arcs and straights, for matching purposes. The space between the quadrupoles and dipoles is occupied by dipole/multipole correctors and chromaticity sextupoles.

A schematic view of a typical arc half-cell assembly is presented in Fig. 1. There are five types of half-cells depending on the quadrupole and correctors' physical dimensions (length and bore radii). This particular half-cell is the most common one and, starting upstream, it consists of: i) a $30 \mathrm{~cm}$-long multi-function corrector, with a $27 \mathrm{~cm}$ bore diameter (27CDM30) having a main dipole coil and windings mounted upon it, capable of producing skewquadrupole and skew-sextupole components; ii) a $40 \mathrm{~cm}-$ long quadrupole, with a $21 \mathrm{~cm}$ bore diameter (21Q40); iii) a $26 \mathrm{~cm}$-long chromaticity sextupole, with a $21 \mathrm{~cm}$ bore diameter; and iv) a $1.2 \mathrm{~m}$-long dipole, with a $17 \mathrm{~cm}$ bore diameter. The distance between the main dipole and quadrupole is being dictated by the optical matching conditions of the arc, the available space and the necessity to accommodate the correctors between them. The correctors have to be as close as possible to the quadrupoles in order to take advantage of the maximum beta functions in these locations [2]. Considering that the magnetic length of the quadrupole is approximately $50 \mathrm{~cm}$ (steel length + bore radius) a "rule of thumb" for estimating the optimal steel-to-steel distance between quadrupole and corrector is by setting it equal to the bore diameter of the quadrupole. By using this simple rule and the available space due to mechanical and vacuum chamber considerations the distance was set to 8 inches $(20.32 \mathrm{~cm})$.

The quadrupole magnet field on a reference radius of $10 \mathrm{~cm}$ and $45^{\circ}$ with respect to the horizontal mid plane

\footnotetext{
* Work performed under the auspices of the US Department of Energy

†papap@bnl.gov
}

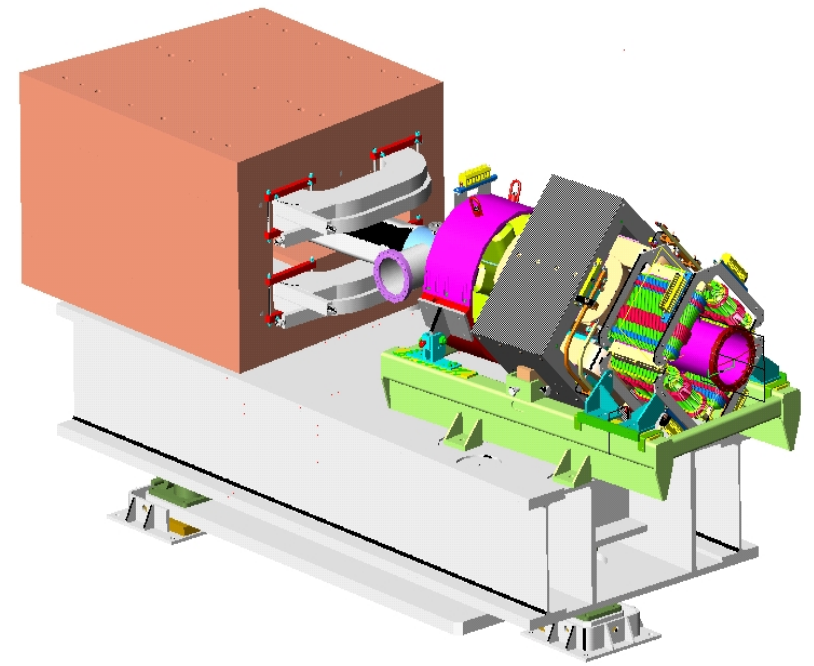

Figure 1: Half-cell schematic layout, with the main dipole, chromaticity sextupole, quadrupole and dipole/skewquadrupole/skew-sextupole corrector.

is presented in Fig. 2. Note that the "spikes" in the field are located exactly at the edge of the quadrupole steel. The magnet fringe field is extending to a large distance and some field lines extend to the location of the corrector steel. It is unavoidably influenced due to the presence of the neighboring corrector. In the same way, the corrector field components interfere with the components of the quadrupole. In this paper, we present the first results of simulations and measurements conducted in order to quantify the magnet field interference between the 21Q40 quadrupole and the 27CDM30 corrector. At a first stage, we were interested to the effect of the corrector on the main quadrupole field component and the influence of the quadrupole on the corrector skew sextupole component.

\section{MAGNETIC FIELD INTERFERENCE}

Magnetic field simulations have being conducted using OPERA-3D [3] for five different configurations of the quadrupole/corrector assembly:

I. 21Q40 quadrupole powered only (without the corrector)

II. 27CDM30 corrector skew sextupole component powered only (without the quadrupole)

III. 27CDM30 corrector skew sextupole component powered in the presence of the quadrupole steel box

IV. 21Q40 quadrupole powered in the presence of the corrector steel box

V. Both magnets are powered (only the skew sextupole of the corrector) 


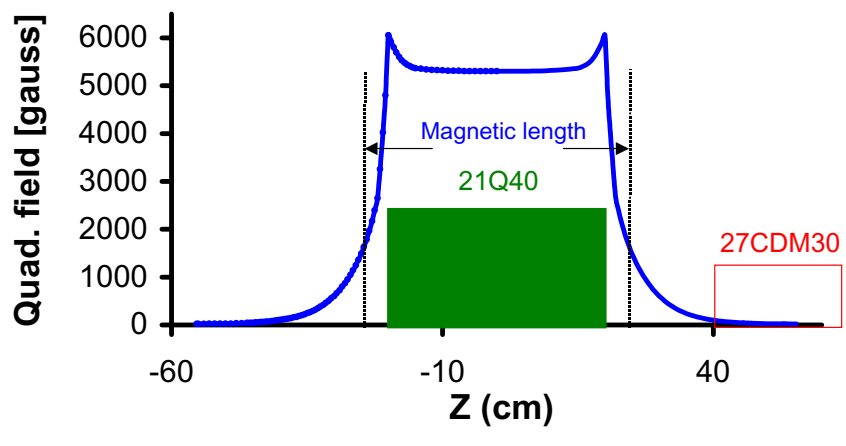

Figure 2: Quadrupole magnetic field of the 21Q40 model computed using OPERA-3D [3] (at $10 \mathrm{~cm}$ radius and $45^{\circ}$ angle). The location of the quadrupole steel and corrector are also drawn (in the simulation only the quadrupole actually exists).

The magnetic field was computed at a radius of $10 \mathrm{~cm}$ and an angle of $45^{\circ}$ from the center of the quadrupole to the center of the corrector. The steel-to-steel distance of the quadrupole and corrector was kept fixed at $20.32 \mathrm{~cm}$. The simulations results for the integrated quadrupole and skew sextupole components of the assembly are shown on Tab. 1 .

Table 1: Integrated quadrupole and skew sextupole field components for the different simulation cases of the 21Q40/27CDM30 assembly.

\begin{tabular}{|l|c|c|}
\hline Cases & $\int b_{2}[\mathbf{G ~ c m}]$ & $\int a_{3}[\mathbf{G ~ c m}]$ \\
\hline I. 21Q40 alone & 130318.9 & 76.1 \\
II. 27CDM30 alone & 0 & -326.7 \\
III. 27CDM30 power. + 21Q40 steel & 0 & -323.3 \\
IV. 21Q40 power. + 27CDM30 steel & 129612.3 & 83.5 \\
V. Both power. & 129612.4 & -231.9 \\
\hline
\end{tabular}

For the $21 \mathrm{Q} 40$ quadrupole, there is a difference of $0.5 \%$ between the nominal integrated field component and the one resulting when both magnets are powered. Note that in the cases where the 21Q40 magnet is not powered, the $b_{2}$ component is zero, as expected by the symmetry of the corrector, which does not allow the existence of normal quadrupole harmonic. On the other hand, when the steel of the corrector exists, the integrated $b_{2}$ is exactly the same as when both magnets powered. This means that the difference to the main quadrupole component results from the interference with the corrector steel box, which distorts slightly the field lines. This effect can be viewed in Fig. 3a where we represent the behavior of the quadrupole fringefield at the vicinity of the 27CDM30 corrector for the three different non-zero cases (I, IV and V). Note that although there are three curves displayed, only two actually can be distinguished. The difference of the field components between the nominal $b_{2}$ field and the field when both magnets are powered is represented in Fig. 3b. The change appears at the edge of the quadrupole field, it takes its maximum somewhere near the corrector steel and then drops gradually inside the body of the corrector.
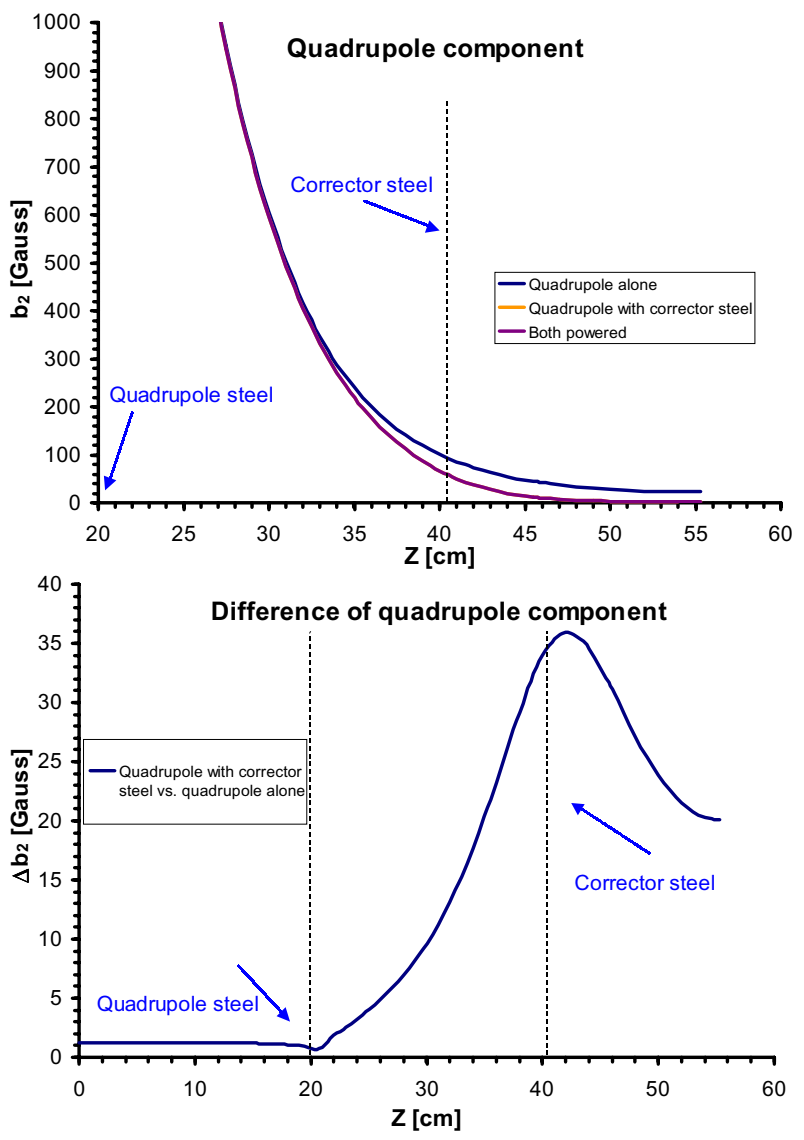

Figure 3: Main quadrupole field component $b_{2}$ of the 21Q40/27CDM30 assembly for the simulation cases I, IV and $\mathrm{V}$ (top) and difference of the field component for the cases I and V (bottom).

The effect of the quadrupole field drop due to the presence of the corrector was also measured, using a long integral coil and moving the corrector to 4 different locations [4]. In Fig. 4, we present the ratio of the measured integrated quadrupole component with respect to the nominal measured field integral (when the magnet stands alone). Note that in the case where the corrector is far enough the field is exactly the same with the nominal case, whereas when the magnet approaches to $20 \mathrm{~cm}$, the magnet looses a few per-mil of its nominal strength, which is compatible with the simulation results.

The effect of the quadrupole core presence to the skewsextupole component of the corrector is somehow different, as in all five cases, there is a non-zero skew sextupole component, even when the corrector does not exist (Tab. 1). This component appears as a geometric error of the quadrupole coil with a positive sign and when it overlaps with the skew-sextupole component of the corrector it reduces the total field to a $29.1 \%$ level. This effect can be also observed in Fig. 5a, where we plot the skew-sextupole component for all simulation cases. Note that now only three of the curves seem to differ, because the influence of the steel boxes is very small. This can be also seen in the Fig. $5 \mathrm{~b}$, where the change of the $a_{3}$ component is plot- 


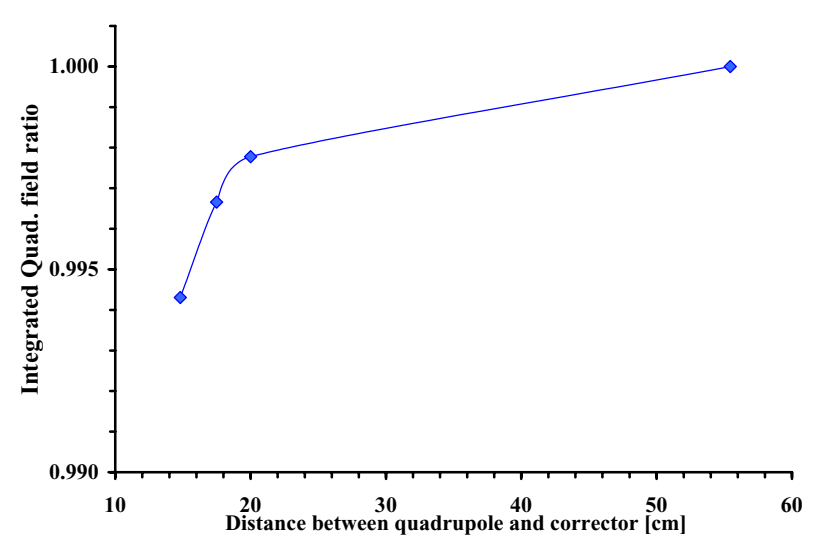

Figure 4: Ratio of measured integrated quadrupole gradient with respect to the nominal gradient, for different distances between the quadrupole and the multi-pole corrector (courtesy of J. Jackson et al. [4]).

ted for the different cases. The difference on $a_{3}$ is minimal with only the presence of the 21Q40 steel whereas it is quite significant when both magnets are powered. However, this effect is not a simple super-position of the skew sextupole field components: by adding the $a_{3}$ components for the cases III and IV, there is still a 3\% difference with respect to the field when both magnets are powered. Without neglecting the possibility of a numerical error (a few gauss is quite small number), one explanation would be that the missing flux has been fed to other field components, allowed by the corrector magnet skew symmetry.

\section{DISCUSSION}

Although the effect of the corrector steel in the quadrupole main field is non-negligible, it can be corrected by the existing quadrupole TRIM windings which are mounted on the core of every quadrupole magnet and are capable of producing $1 \%$ of the main coil gradient. This effect should be also taken into account as a systematic error in the current read-outs when powering the magnet during operation. On the other hand, for the skew-sextupole component there is not an evident solution. The idea of introducing mirror plates at the edge of the magnets in order to isolate their fringe fields was quickly dropped due to the potential compromise of the quadrupole field quality. At this stage of the design, where most of the magnets are in the production phase, the preferred action is to improve the geometric field quality of the quadrupole, in order to minimize further the skew-sextupole error, thus eliminating its overlapping with the $a_{3}$ component of the corrector.

Let us mention that the effect of interference of the magnet steel, was also observed to all the normal error harmonics computed (up to dodecapole $b_{6}$ ) and to the three skew error harmonics $a_{2}, a_{4}$ and $a_{6}$. These harmonics do not appear in the corrector field expansion, when it is powered alone. On the other hand, there was an overlapping of the remaining skew harmonics computed $a_{1}, a_{5}$ field as for the skew sextupole component, as they are aloud by the cor-
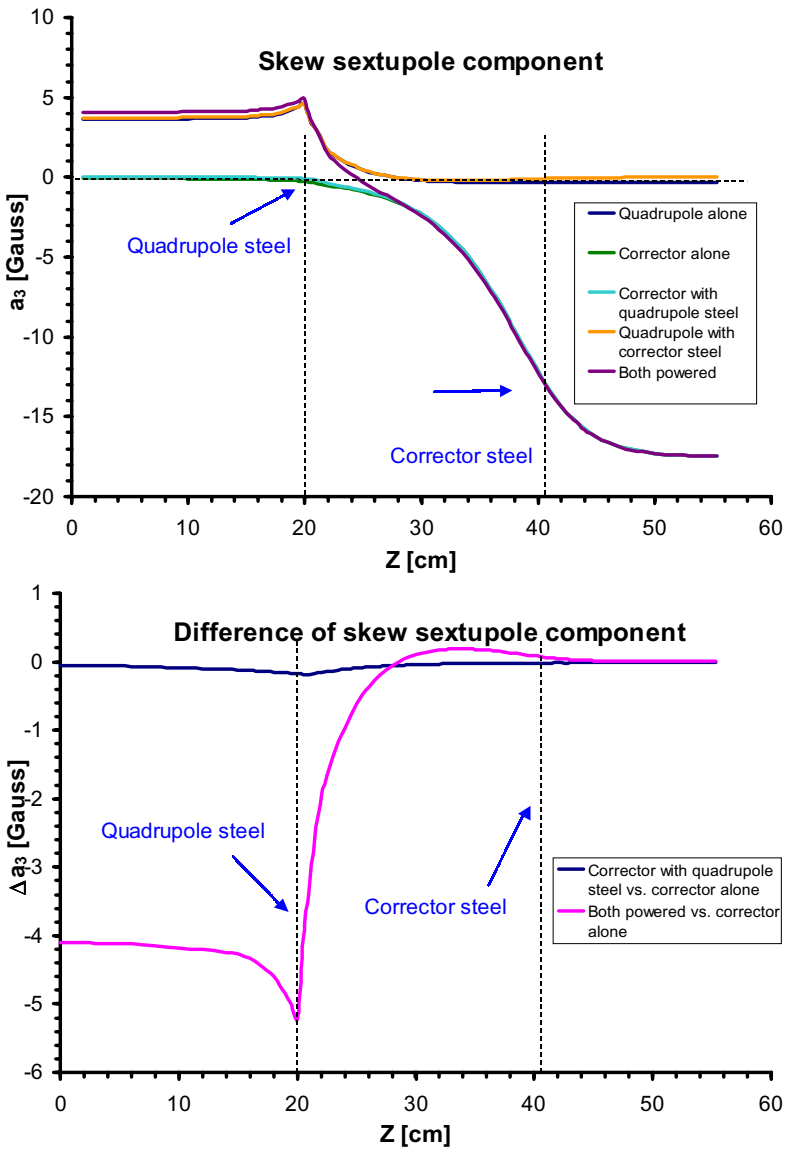

Figure 5: Skew sextupole field component of the 21Q40/27CDM30 assembly for all the simulation cases (top) and difference of the field component between the cases I and V (bottom).

rector geometry. The clear message given by the very first results of this study is that in high-intensity low-energy, with short, wide magnets, it is necessary to investigate and quantify the effect of fringe-field interference, in opposition with the usual procedure of modeling the magnets as stand alone units. Indeed, the effect, although small in the case of the quadrupole main field, is non-negligible for the skew sextupole component of the corrector so as to compromise the performance of the correction schemes. Our perspectives are to proceed with this study for investigating the influence to the other field components of the corrector. Finally, it would be interesting to extend it to the multi-pole low-field sextupole and octupole correctors as well as the chromaticity sextupoles of the SNS ring.

We would like to thank N. Tsoupas and J. Wei, for providing useful suggestions and, J. Jackson and C. Spataro for performing the magnet measurements.

\section{REFERENCES}

[1] J. Wei, et al., PRST-AB, 3:080101, 2000.

[2] C.J. Gardner, BNL/SNS Technical Note 40, 1997.

[3] Vector Fields, Ltd, Oxford England.

[4] J. Jackson et al., BNL AM-MD-304, in preparation. 